\title{
Erratum to: Fluorescent Dye Labeled Iron Oxide/Silica Core/Shell Nanoparticle as a Multimodal Imaging Probe
}

Eue Soon Jang • Seung Yong Lee • Eui-Joon Cha • In-Cheol Sun • Ick Chan Kwon • Dukjoon Kim • Young II Kim • Kwangmeyung Kim • Cheol-Hee Ahn

Published online: 2 December 2014

(C) Springer Science+Business Media New York 2014

\section{Erratum to: Pharm Res}

\section{DOI I0.1007/s| |095-0|4-|426-z}

The affiliations for two authors are incorrect in the published article. I. C. Kwon should be listed in the third affiliation rather than the fourth affiliation. D. Kim should be listed in the fourth affiliation rather than the fifth affiliation. The correct affiliations are shown in this erratum.

The online version of the original article can be found at http://dx.doi.org/l0. | 007/s | | 095-0|4-| 426-z.

E. S. Jang

Advanced Materials Educational Institute, Kumoh National Institute of

Technology, Sanho-ro 77, Yangho, Gumi 730-70I, South Korea

\section{S. Y. Lee • E.-J. Cha • C.-H. Ahn}

Research Institute of Advanced Materials (RIAM), Department of

Materials Science and Engineering, Seoul National University

San 56- I, SillimGwanak, Seoul I I I-744, South Korea

I.-C. Sun •I. C. Kwon· K. Kim

Biomedical Research Center, Korea Institute of Science and Technology (KIST), 39-I, HawolgokSeongbuk, Seoul I36-79I, South Korea

\section{Kim}

Department of Chemical Engineering, Theranostic Macromolecule Research Center, Sungkyunkwan University, Suwon,

Kyunggi 440-746, South Korea

\section{Y. I. Kim}

Department of Radiology, Seoul National University Hospital

28, Yeongeonjongno, Seoul II0-744, South Korea

\section{C.-H. Ahn $(\bowtie)$}

Materials Science and Engineering, Seoul National University, Seoul 151-744, South Korea

e-mail: chahn@snu.ac.kr

K. Kim $(\square)$

Biomedical Research Center, Korea Institute of Science and Technology, Seoul I36-79|, South Korea

e-mail: kim@kist.re.kr

Y. I. $\operatorname{Kim}(\square)$

Radiology, Seoul National University Hospital, Seoul I I0-744,

South Korea

e-mail: kyimd@snu.ac.kr 